particles are so large that gain fluctuations from their discreteness is not appreciable.

It is to be remarked that because of inclusion of fluctuation effects, the quantum model is more complete than the classical nonlinear circuit. The idealized reactor is noise-free because its response is a single valued function of stimulus. The noise sources inherently associated with any physical reactor must be evaluated from the departure from the ideal. These departures include the dissipative effects which any real inductor or capacitor exhibits. The low-noise performance of the back-biased semiconductor junction used as a nonlinear capacitor arises from the relatively small dissipation of energy.

The final difference which we wish to mention is perhaps the most puzzling of all. This is that the idealized reactor is described by nonlinear equations while the analogous quantum mechanical system is analyzed by linear operations. It is true that we do not solve for the same quantities in both cases. In the reactor, we calculate relations between current, voltage, charge, and flux linkage. In the maser, Schroedinger and Heisenberg's methods actually evaluate probability density functions from which only average values of observable quantities can be obtained. But the calculations are linear, and for large systems the average values furnish an adequate description.

\section{REFERF:NCES}

[1] C. H. Page, "Frequency conversion with positive nonlinear resistors," J. Res. NBS, vol. 56, pp. 179--182; April, 1956.

2] L. Kuhn, "veber ein neues Radiotelephonisches System," Archiv fur Elektrotech., Band 4, Heft 29, pp. 816-819; July 16, 1914 .

[3] E. F. W. Alexanderson and S. P. Nixdorff, "A magnetic amplifier for radio telephony," Proc. IRE, vol, 4, pp. 101-120; April, 1916.

[4] J. M. Manley and E. Peterson, "Negative resistance effects in saturable reactor circuits," Trans. AIEE, vol. 65, pp. 870881; December, 1946.

[5] J. M. Manley, "Some general properties of magnetic amplifiers," Proc IRE, vol. 39, pp. 242-251; March, 1951.

[6] W. R. Bennett, "New results in the calculation of modulation products," Bell Syst. Tech. J., vol. 12, pp. 228-243; April, 1933.

[7] J. M. Manley and H. E. Rowe, "Some general properties of nonlinear elements, Part I, General energy relations," Proc. IRE, vol. 44, pp. 904-913; July, 1956.

[8] C. H. Page, "Frequency conversion with nonlinear reactance," $J$. Res. NBS, vol. 58, pp. 227-236; May, 1957.

[9] M. 'T. Weiss, "Quantum derivation of energy relations analogous to those for nonlinear reactance," Proc. IRE, vol. 45, pp. 1012-1013; July, 1957.

[10] J. R. Pierce, "Use of the principles of conservation of energy and momentum in connection with the operation of wave-type parametric amplifiers," J. Appl. Phys., vol. 30, pp. 1341-1346; September, 1959.

[11] J. M. Manley and H. E. Rowe, "General energy relations in nonlinear reactances," Proc. IRE, vol. 47, pp. 2115-2116; December, 1959.

[12] W. R. Bennett, "Electrical Noise," McGraw-Hill Book Co., Inc., New York, N. Y.; 1961.

[13] K. Shimoda, H. Takahasi, and C. H. Townes, "Fluctuations in amplification of quanta," J. Phys. Soc. Japan, vol. 12, pp. 686-700; June, 1957.

\title{
The Transient Behavior of Nonlinear Systems*
}

\author{
FRANCIS H. CLAUSER $\dagger$
}

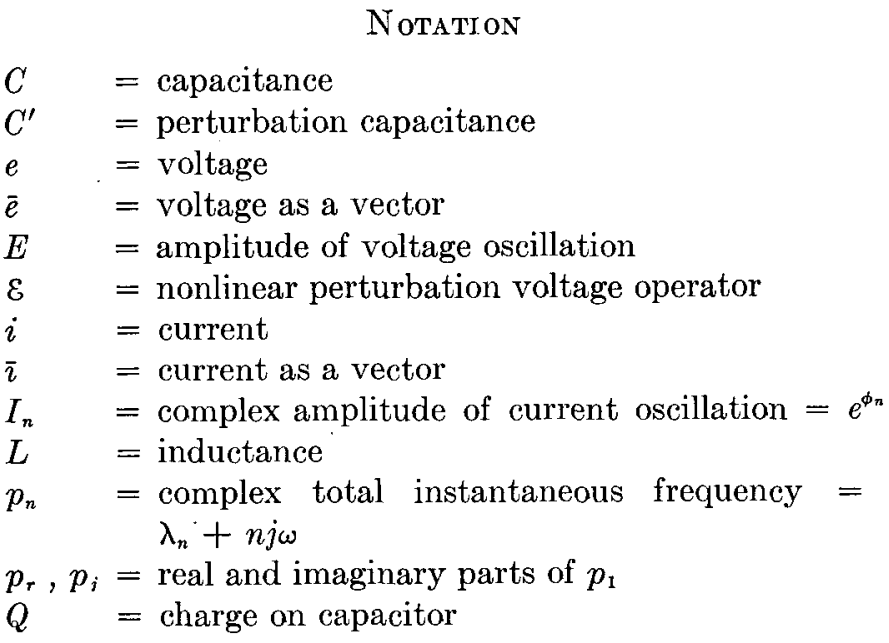

* Received by the PGCT, May 16, 1960. This research was supported by the United States Air Force through the Air Force Office of Scientific Res. of the Air Res. and Dev. Command, under Contract Number AF 49(638)-496.

$\dagger$ The Johns Hopkins University, Baltimore, Md.

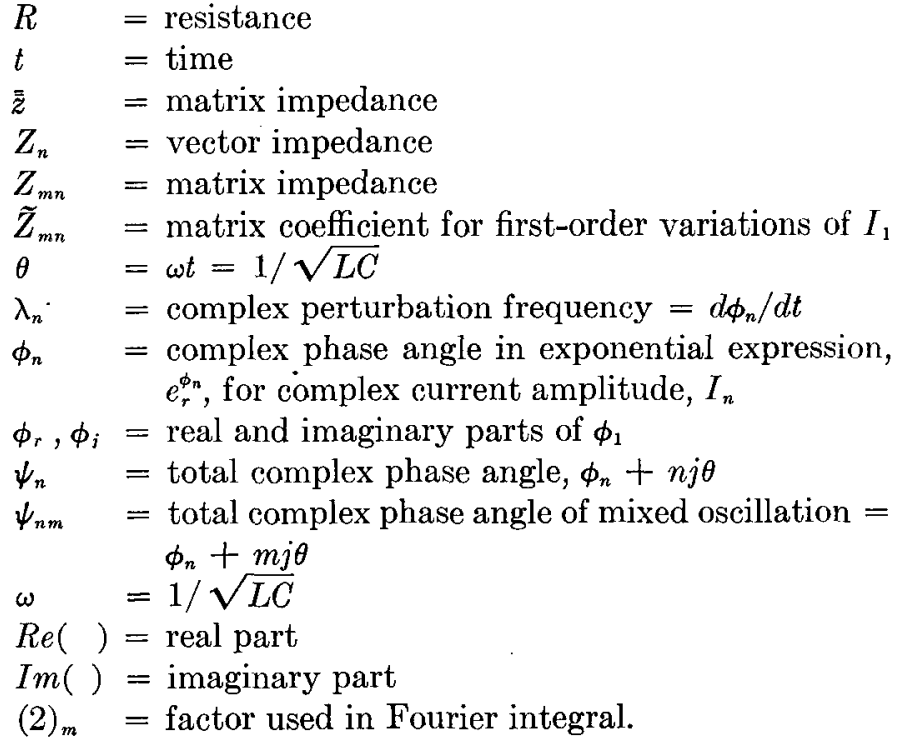

Subscripts denote harmonic component.

Superscripts denote order of magnitude. 


\section{INTRODUCTION}

T $\mathrm{N}$ an earlier paper, ${ }^{1}$ the author presented a method of treating nonlinear systems by means of nonlinear impedances which, in addition to depending on the frequencies, also depend upon the amplitudes of the oscillations taking place in the circuit. When a number of frequencies are present, as is invariably the case in nonlinear systems, such impedances are not scalars, but rather are matrices, with the matrix components corresponding to the different frequencies. In this earlier paper, it was shown that the method can treat a wide variety of problems, such as subharmonic and superharmonic resonance, oscillator lock-in, parametric amplification, super-regeneration, rectification, modulation and the like. Attention was devoted largely to steady-state behavior. In the present paper, emphasis will be placed on the transient behavior of nonlinear systems.

\section{Classical Perturbation Theory}

We begin our analysis by considering the general features of the behavior of nonlinear systems as revealed by the elassical perturbation theory of Poincaré. This theory serves as the basis for most of the perturbation schemes that deal with oscillatory phenomena in all the different branches of science. When formulated in circuit theory terms, it may be illustrated by the following example.

Suppose we start with an $L-C$ circuit described by the canonical differential equation for a sinusoid of a single frequency, $\omega$ :

$$
L \frac{d i}{d t}+\frac{1}{C} \int i d t=0 \text { where } \omega=\frac{1}{\sqrt{L C}} .
$$

Into this circuit we insert one or more elements (see Fig. 1) whose valtage, $e$, is related to the current, $i$, by the operator, $\varepsilon$; thus, $e=\varepsilon(i)$. Here, $\varepsilon$ may contain such operations as differentiation, integration, time delay and the like in nonlinear combination with $i$ itself.

When such perturbing elements are placed in the circuit, the following interactions will occur. The original current, $i^{0}(t)$ flowing through the elements will create a perturbing voltage, $\varepsilon\left(i^{0}\right)$. This voltage will produce a change, $i^{-1}(t)$, in the current flowing through the circuit. This altered current, $i^{0}+i^{1}$, will in turn produce a change $\varepsilon\left(i^{0}, i^{1}\right)=$

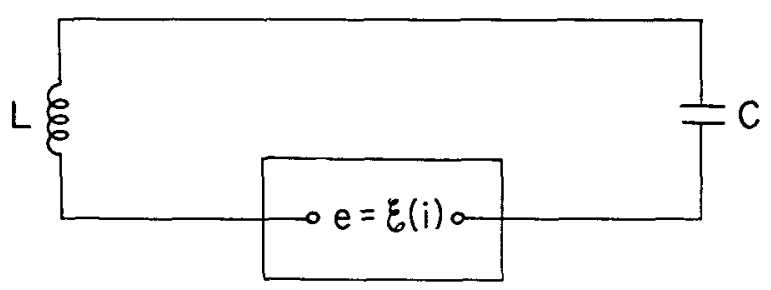

Fig. 1-Simple $L C$ circuit containing perturbing nonlinear elements.

${ }^{1} \mathrm{~F}$. H. Clauser, "A theory of nearly linear systems," in "Proceedings of the Congress of the International Federation of Automatic Control, Moscow, USSR," Butterworth Scientific Publications, Inc., London, England; 1960. $\varepsilon\left(i^{0}+i^{1}\right)-\varepsilon\left(i^{0}\right)$ in the voltage across the elements. We may express this set of interactions by the sequences:

$$
\begin{aligned}
& i(t)=i^{0}(t)+i^{1}(t)+i^{2}(t)+\cdots \\
& \varepsilon(i)=\varepsilon\left(i^{0}\right)+\varepsilon\left(i^{0}, i^{1}\right)+\varepsilon\left(i^{0}, i^{1}, i^{2}\right)+\cdots .
\end{aligned}
$$

Clearly, each change in $\varepsilon$ depends upon the characteristics of the elements and each change in $i$ depends upon the characteristics of the original circuit. Because of interaction, the progression of changes in $\varepsilon$ and $i$ depend upon both the elements and the circuit in which the elements are placed. This progression is described by the set of circuit equations:

$$
\begin{aligned}
& L \frac{d i^{0}}{d t}+\frac{1}{C} \int i^{0} d t=0 \\
& L \frac{d i^{1}}{d t}+\frac{1}{C} \int i^{1} d t=-\varepsilon\left(i^{0}\right) \\
& L \frac{d i^{2}}{d t}+\frac{1}{C} \int i^{2} d t=-\varepsilon\left(i^{0}, i^{1}\right) .
\end{aligned}
$$

Since the nonlinear term of each equation involves only $i$ 's that have been determined from preceding equations, the set may be solved seriatim.

\section{The Form of the Perturbation Solutions}

At first glance, one might think that the form of the solutions obtained by this perturbation procedure would depend upon the operator, $\varepsilon(i)$. However, this is not the case. This is most easily shown by means of a few examples.

First, let us see what happens when the perturbing element is simply a linear capacitor of low impedance. If the capacity of this perturbing element is $C^{\prime}$, then $\mathcal{E}(i)=1 / C^{\prime} \int i d t$ and the circuit equation becomes

$$
L \frac{d i}{d t}+\frac{1}{C} \int i d t=-\frac{1}{C^{\prime}} \int i d t
$$

Correspondingly, the set of perturbation equations becomes

$$
\begin{aligned}
& L \frac{d i^{0}}{d t}+\frac{1}{C} \int i^{0} d t=0 \\
& L \frac{d i^{1}}{d t}+\frac{1}{C} \int i^{1} d t=-\frac{1}{C^{\prime}} \int i^{0} d t \\
& L \frac{d i^{2}}{d t}+\frac{1}{C} \int i^{2} d t=-\frac{1}{C^{\prime}} \int i^{1} d t .
\end{aligned}
$$

The solution for the first equation is $i^{0}=I^{0} e^{i \omega t}$ where $\omega=1 / \sqrt{L C}$. When this is placed in the operator on the right of the second equation, it yields a resonant input which is an eigenfunction of the operator on the left. This leads to a solution for $i^{1}$ containing the secular term, $t e^{j \omega t}$. Correspondingly, successive equations will 
yield terms containing $t^{2} e^{i \omega t}, t^{3} e^{i \omega t}$, etc. The final solution for $i$ is, in real form,

$$
\begin{aligned}
i=I^{0}\{[1- & \left.\frac{\omega^{2} t^{2}}{8}\left(\frac{C}{C^{\prime}}\right)^{2}\left(1-\frac{1}{2} \frac{C}{C^{\prime}} \cdots\right)+\cdots\right] \cos \omega t \\
& +\left[-\frac{\omega t}{2}\left(\frac{C}{C^{\prime}}\right)\left(1-\frac{1}{4} \frac{C}{C^{\prime}} \cdots\right)\right. \\
& \left.\left.+\frac{\omega^{3} t^{3}}{48}\left(\frac{C}{C^{\prime}}\right)^{3}\left(1-\frac{3}{4} \frac{C}{C^{\prime}} \cdots\right)-\cdots\right] \sin \omega t\right\} .
\end{aligned}
$$

In this simple case, we are also able to obtain the exact solution:

$$
i=I^{0} \cos \left(\omega t \sqrt{1+\frac{C^{\prime}}{C^{\prime}}}\right) .
$$

This may be written in the form

$$
i=I^{0}[A(t) \cos \omega t+B(t) \sin \omega t]
$$

where the two coefficients

$$
\begin{aligned}
& A(t)=\cos \omega t\left(\sqrt{1+\frac{C}{C^{\prime}}}-1\right) \\
& B(t)=-\sin \omega t\left(\sqrt{1+\frac{C}{C^{\prime}}}-1\right)
\end{aligned}
$$

vary slowly because of the smallness of the factor $\left(\sqrt{1+C / C^{\prime}}-1\right)$.

Comparing these two solutions, we get our first glimpse of the way in which the perturbation procedure expresses its results. It has ground out a power series in terms of the small quantity, $C / C^{\prime}$, for the coefficients, $A$ and $B$, of the basic oscillatory terms. But what is of greater importance to us is the fact that in so doing, it has also ground out power series expansions in the time for the slow variations of these coefficients which serve to change the frequency of the oscillation from

$$
\frac{1}{\sqrt{L C}} \text { to } \sqrt{\frac{1}{L}\left(\frac{1}{C}+\frac{1}{C^{\prime}}\right)} \cdot
$$

Thus in the perturbation method, we end up with oscillatory solutions whose coefficients are slowly varying power series in the time.

Next, let us see what happens when we use a linear resistor as a perturbing element. In this case, we have $\varepsilon(i)=-R i$, and, if we carry out the same procedure, we obtain the perturbation solution:

$$
\begin{aligned}
i=I^{0}\{[1- & \frac{\omega t}{2}\left(R \sqrt{\frac{C}{L}}\right) \\
& \left.+\frac{\omega^{2} t^{2}}{8}\left(\frac{R^{2} C}{L}\right)\left(1-\frac{R^{2} C}{16 L}\right) \cdots\right] \cos \omega t \\
& \left.-\left[\frac{\omega t}{8}\left(\frac{R^{2} C}{8 L}\right)-\frac{\omega^{2} t^{2}}{16}\left(R \sqrt{\frac{C}{L}}\right)^{3} \cdots\right] \sin \omega t\right\} .
\end{aligned}
$$

We also are able to obtain the exact solution,

$$
i=I^{0} e^{-R t / 2 L} \cos \left(t \sqrt{\frac{1}{L C}-\frac{R^{2}}{4 L^{2}}}\right),
$$

$$
\begin{aligned}
& \text { which, for the purpose of comparison, can be written: } \\
& \begin{aligned}
i=I^{0}\left\{\left[e^{-R t / 2 L} \cos t\left(\sqrt{\frac{1}{L C}-\frac{R^{2}}{4 L^{2}}}-\frac{1}{\sqrt{L C}}\right)\right] \cos \omega t\right. \\
\left.-\left[e^{-R t / 2 L} \sin t\left(\sqrt{\frac{1}{L C}-\frac{R^{2}}{4 L^{2}}}-\frac{1}{\sqrt{L C}}\right)\right] \sin \omega t\right\} .
\end{aligned}
\end{aligned}
$$

In this case, the resistor causes the current to decay exponentially. It also changes the frequency of the basic oscillation from

$$
\frac{1}{\sqrt{L C}} \text { to } \sqrt{\frac{1}{L C}-\frac{R^{2}}{4 L^{2}}} .
$$

We see that the perturbation method expresses these changes as power series expansions in time for the slowly varying exponential and trigonometric coefficients of the basic oscillatory terms. Hence, we have the same pattern as before for the perturbation solution, namely , oscillatory terms whose coefficients are slowly varying power series in the time.

Now that we have explored how the perturbation procedure expresses its results for linear elements, let us see how it works for nonlinear elements. We consider the well-known equation of van der Pol:

$$
\frac{d^{2} y}{d t^{2}}-\alpha\left(1-y^{2}\right) \frac{d y}{d t}+y=0 .
$$

This equation can be integrated once and rewritten in the form

$$
\frac{d y}{d t}+\int y d t=\alpha y\left(1-y^{3} / 3\right)
$$

In circuit terminology, this then takes on the form

$$
L \frac{d i}{d t}+\frac{1}{C} \int i d t=R i\left(1-\frac{\beta i^{2}}{3}\right),
$$

which, in the circuit of Fig. 1, corresponds to a perturbing element having

$$
-\varepsilon(i)=R i-\frac{\beta R i^{3}}{3}
$$

i.e., a nonlinear resistor which has a cubic relation between voltage and current and which exhibits a negative resistance when the current is small. For this case, the perturbation equations become:

$$
\begin{aligned}
& L \frac{d i^{0}}{d t}+\frac{1}{C} \int i^{0} d t=0 \\
& L \frac{d i^{1}}{d t}+\frac{1}{C} \int i^{1} d t=R i^{0}-\frac{\beta R}{3}\left(i^{0}\right)^{3} \\
& L \frac{d i^{2}}{d t}+\frac{1}{C} \int i^{2} d t=R i^{1}-\beta R i^{1}\left(i^{0}\right)^{2} \\
& L \frac{d i^{3}}{d t}+\frac{1}{C} \int i^{3} d t=R i^{2}-\beta R\left[i^{2}\left(i^{0}\right)^{2}+\left(i^{1}\right)^{2} i^{0}\right] .
\end{aligned}
$$


When solved, this set of equations yields the result:

$$
\begin{aligned}
i= & I^{0} \cos \omega t\left\{1+\omega t\left[R \sqrt{\frac{C}{L}}\left(\frac{1}{2}-\frac{\beta I^{0^{2}}}{8} \cdots\right)\right.\right. \\
& \left.+(R \sqrt{C})^{3}\left(\frac{1}{32}-\frac{\beta I^{0^{2}}}{16}+\frac{43 \beta^{2} I^{0^{4}}}{1024} \cdots\right) \cdots\right] \\
& \left.+\omega^{2} t^{2}\left[R^{2} \frac{C}{L}\left(\frac{1}{8}-\frac{\beta I^{0^{2}}}{8}+\frac{3 \beta^{2} I^{04}}{128} \cdots\right)+\cdots\right]+\cdots\right\} \\
& +I^{0} \sin \omega t\left\{\omega t\left[R^{2} \frac{C}{L}\left(\frac{1}{8}-\frac{\beta I^{0^{2}}}{8} \cdots\right) \cdots\right]\right. \\
& \left.+\omega^{2} t^{2}\left[\left(R \sqrt{\frac{C}{L}}\right)^{3}\left(\frac{3}{32}-\frac{\beta I^{03}}{2} \cdots\right) \cdots\right] \ldots\right\} \\
& +\beta I^{0^{3}} \cos 3 \omega t\left\{R^{2} \frac{C}{L}\left(-\frac{1}{16}+\frac{\beta I^{0^{2}}}{64} \cdots\right)\right. \\
& \left.+\omega t\left[R^{2} \frac{C}{L}\left(-\frac{35}{1024}+\frac{65 \beta I^{02}}{4096} \cdots\right) \cdots\right] \cdots\right\} \\
& +\beta I^{0^{3}} \sin 3 \omega t\left\{-\frac{1}{32}+R^{2} \frac{C}{L}\left(\frac{97}{4096}+\frac{185 \beta I^{0^{2}}}{16384} \cdots\right)\right. \\
& \left.+\omega t\left[R \sqrt{\frac{C}{L}}\left(-\frac{3}{64}+\frac{3 \beta I^{02}}{256} \cdots\right) \cdots\right] \ldots\right\} \\
& +\beta^{2} I^{0^{3}} \cos 5 \omega t\left\{-\frac{1}{3072}\right. \\
& \left.+\omega t\left[R \sqrt{\frac{C}{L}}\left(-\frac{17}{6144}+\frac{17 \beta I^{0^{2}}}{24576} \cdots\right) \cdots\right] \ldots\right\} \\
& +\beta^{2} I^{0^{\circ}} \sin 5 \omega t\left\{R \sqrt{\frac{C}{L}}\left(-\frac{25}{73728}+\frac{10 \beta I^{0^{2}}}{73728} \cdots\right) \cdots\right\} \\
& +e t c .
\end{aligned}
$$

Now it is well known that nonlinear elements can generate higher harmonics of the basic oscillation (and a de component as well). And quite properly in this example, in contrast to the preceding linear cases, we see such terms appearing in the solution.

However, we see in addition a phenomenon which is not characteristic of the response of an isolated element to an oscillatory input, namely the appearance of slowly varying amplitudes for the oscillatory terms. Just as in the linear case, these slow variations result from the interaction of the element with the circuit in which the element is placed. But now we see that in the nonlinear case, the coefficients of the harmonics as well as the fundamental oscillation vary slowly with the time. Furthermore, we see that the perturbation procedure typically expresses these slowly varying coefficients as power series in the time.

In summary, we now see that the general form for the perturbation solution will be as follows. First, the solution will contain the dominant oscillatory term which is characteristic of the linear operator on the left side of the equation. Second, it will contain all of the appropriate harmonics (and de term) that one would expect nonlinearity to generate from the dominant oscillation. Third, all of the amplitudes of these oscillatory terms will be expressed as slowly varying power series in the time.
Traditionally, this classical perturbation procedure has been faced with the following inconsistency which has proved to be quite troublesome. The oscillatory terms give the short-term or local pattern of behavior of the system. Once we have digested the short-term wave form, we begin to look at the long-term behavior of the system. This is given to us by the slowly varying amplitudes of the oscillatory terms. Consistent with the fact that these amplitudes carry the information about the long-term behavior of the system, one would like for their solutions to have long-term validity. However, the perturbation procedure quite inconsistently expresses these long-term functions as power series in the time, a mode of expression which is notoriously poor for longterm validity.

It is for this reason that we set as the first of the goals for the present paper the problem of obtaining perturbation solutions that will have long-term validity for the slowly varying coefficients that will be consistent with the role they play in giving the long-term behavior of the system.

\section{Operational Calculus for NonLinear Oscillations}

There appears to be little in common between the nonlinear perturbation procedure which we have just discussed and the operational calculus which is so commonly used to solve linear circuit problems. In the present section, we shall show that there is more common ground than appears at first glance. We shall also show what changes are necessary in the operational calculus when we are dealing with moderately nonlinear systems.

From the perturbation procedure, we have seen that the behavior of the nonlinear system described by

$$
L \frac{d i}{d t}+\frac{1}{C} \int i d t=\varepsilon(i)
$$

can be expressed in the form

$$
i=I_{0}+I_{1} e^{i \omega t}+I_{2} e^{2 i \omega t}+I_{3} e^{3 j \omega t}+\text { etc. }
$$

where the amplitude, $I_{0}, I_{1}, I_{2}$, etc. are slowly varying functions of the time.

A logical next step is to express the amplitudes themselves as complex exponentials. Thus we write

$$
i=e^{\psi_{0}}+e^{\psi_{1}}+e^{\psi_{1}}+e^{\psi_{3}}+\text { etc. }
$$

Here we have put $\psi_{n}=\phi_{n}+n j \theta$ where $\theta=\omega t$ and $e^{\phi}{ }^{\phi}=I_{n}$. The $\phi n$ 's are complex. Their real parts give the slowly varying exponential amplitudes of the current components. Their imaginary parts give the slowly varying phase angles of these current components. The $\mathrm{dc}$ component, $\psi_{0}\left(=\phi_{0}\right)$, is purely real.

Since the phase angles of the current components vary with the time, the time derivatives of these phase angles will correspond to perturbations in the frequencies of the components. Correspondingly, we may view time derivatives of the exponential amplitudes as Napierian or 
exponential frequencies. We may express this as follows. If we put $\omega=d \theta / d t=1 / \sqrt{L C}$, this becomes the basic frequency of the system. Also, if we put

$$
\lambda_{n}=\frac{d \phi_{n}}{d t} \quad \text { and } \quad p_{n}=\frac{d \psi_{n}}{d t}=\frac{d \phi_{n}}{d t}+n j \frac{d \theta}{d t}=\lambda_{n}+n j \omega,
$$

we see that the $p_{n}$ 's are the total complex "instantaneous" frequencies of the system. They consist of the dominant trigonometric frequencies, $n j \omega$, and the complex frequency perturbations, $\lambda_{n}$. These latter consist of real parts which are the slowly varying exponential frequencies and imaginary parts which are those portions of the trigonometric frequencies which are slowly varying.

Since we are dealing with predominantly oscillatory phenomena, the fundamental trigonometric frequency and its harmonics all have large (zero-order) fixed parts. Both the trigonometric and exponential frequencies have first-order perturbations from these large, purely imaginary parts. These first-order perturbations vary slowly with the time in such a way that the frequency derivatives, $d p_{n} / d t$, are quantities of second order; $d^{2} p_{n} / d t^{2}$ are of third order, etc.

Let us now examine what happens to operational procedures when we use such slowly varying instantanecus frequencies. For a typical term it is easy to verify that if

$$
y=e^{\psi} \quad \text { and } \quad p=\frac{d \psi}{d t},
$$

then

$$
\begin{aligned}
\frac{d y}{d t} & =p y \\
\frac{d^{2} y}{d t^{2}} & =\left(p^{2}+\frac{d p}{d t}\right) y \\
\frac{d^{3} y}{d t^{3}} & =\left(p^{3}+3 p \frac{d p}{d t}+\frac{d^{2} p}{d t^{2}}\right) y \\
\frac{d^{4} y}{d t^{4}} & =\left[p^{4}+6 p^{2} \frac{d p}{d t}+3\left(\frac{d p}{d t}\right)^{2}+4 p \frac{d^{2} p}{d t^{2}}+\frac{d^{3} p}{d t^{3}}\right] y
\end{aligned}
$$

and so on for higher derivatives.

Also,

$$
\begin{aligned}
\int y d t=\left\{\frac{1}{p}\right. & \left.-\frac{1}{p} \frac{d}{d t}\left(\frac{1}{p}\right)+\frac{1}{p} \frac{d}{d t}\left[\frac{1}{p} \frac{d}{d t}\left(\frac{1}{p}\right)\right] \cdots\right\} y \\
& =\left[\frac{1}{p}+\frac{d p / d t}{p^{3}}+\frac{3}{p^{5}}\left(\frac{d p}{d t}\right)^{2}-\frac{d^{2} p / d t^{2}}{p^{4}} \cdots\right] y
\end{aligned}
$$

and similar expressions for higher integrals.

We notice that when the frequencies are variable, we are no longer privileged to replace $d / d t$ by $p$. In this form, the operational calculus no longer offers the possibility of substituting a simple algebra of $p$ for the complexities of differential equations involving $d / d t$. Instead, it leads to the combined difficulties of both spectral analysis and differential equations, involving derivatives of the frequencies.

We notice that the deviations from the simple operational scheme of replacing $d / d t$ by $p$ involve terms that are of second order and higher. It is for this reason that first-order theories such as the method of equivalent linearization and the method of the describing function can continue to use the conventional operational procedures.

However, we know that a great number of nonlinear phenomena can not be described by a first-order analysis which, of necessity, takes into account only relatively simple interactions. If we are to cope with the truly challenging problems of nonlinear systems, we must be able to include higher-order terms in our analysis. Consequently we set as the second goal of the present paper the problem of obtaining a workable method of operational analysis that will be valid to the higher orders of accuracy.

The problem of replacing the derivatives of $p_{n}$ by purely algebraic expressions such that all operational procedures will become simply algebraic manipulations must be postponed until we consider the behavior of the entire circuit, since this replacement involves nonlinear interactions taking place in the complete circuit. At that time, we shall indeed find a way eliminating the derivatives of $p_{n}$ such that purely algebraic procedures may be used. Assuming for the moment that such an answer will be forthcoming, we see that for linear elements such as inductors, resistors, capacitors and the like, which involve differentiation and integration, we can extend the usual concepts of impedance and admittance to cover the case of a current or voltage having a single harmonic component whose amplitude and frequency are slowly varying. In this case, the impedance itself will be slowly varying.

\section{The Concept of Matrix Impedance}

In a circuit containing nonlinear elements, it is inevitable that a number of different frequencies will be present. When more than one frequency is present, even in a linear circuit, the concept of a scalar impedance breaks down. For example, when a current containing the two frequencies, $\omega_{1}$ and $\omega_{2}$, passes through an inductor, one cannot say the inductor has an impedance, $j \omega L$. In reality, it has two impedances, $j \omega_{1} L$ and $j \omega_{2} L$, each being used separately for its own oscillation.

Suppose we were to think of a multifrequency current as being a vector,

$$
\bar{\imath}=I_{1} e^{i \theta_{1}}+I_{2} e^{i \theta_{3}}+I_{3} e^{i \theta_{3}} \cdots,
$$

whose components $I_{1}, I_{2}, I_{3}$, etc. are the factors which multiply the unit vectors $e^{j \theta_{2}}, e^{j \theta_{2}}, e^{j \theta_{3}}$, etc. We also think of the corresponding voltage,

$$
\bar{e}=E_{1} e^{j \theta_{2}}+E_{2} e^{j \theta_{2}}+E_{3} e^{j \theta_{3}} \ldots
$$

as being a vector. In this language, the impedance becomes a matrix, $\overline{\bar{z}}$ or $Z_{n n}$, which relates the voltage to the current:

$$
\bar{e}=\overline{\bar{z}} \cdot \bar{\imath} \quad \text { or } \quad E_{m}=\sum_{n} Z_{m n} I_{n} .
$$

For the usual linear devices, $\overline{\bar{z}}$ is a diagonal matrix, since a current at one frequency can induce a voltage only at the same frequency. Thus, for a linear inductor 
with two frequencies present, $Z_{11}=j \omega_{1} L, Z_{22}=j \omega_{2} L$, $Z_{12}=Z_{21}=0$. In the next section, we shall see how the impedance matric can be used for nonlinear devices.

\section{Frequency Behavior of Nonlinear Elements}

Let us now look at the frequency behavior of nonlinear elements such as those represented by the nonlinear operator, $\varepsilon(i)$. Since such elements represent perturbations in the circuit, the greatest terms that occur will be of first order.

These largest terms will result from the action of the dominant current, ${ }^{2} I_{1} e^{i \theta}$, in flowing through the nonlinear device. In obtaining these first-order terms, we are to treat $I_{1}$ as a purely real constant.

We can express this first-order result in the form $\varepsilon\left(I_{1} \cos \theta\right)=E_{0}+E_{1} \cos \left(\theta+\alpha_{1}\right)+E_{2} \cos \left(2 \theta+\alpha_{2}\right) \cdots$ or alternatively, after the nonlinear operations have been performed, we may revert to the complex notation:

$$
\varepsilon=E_{0}+E_{1} e^{i \theta}+E_{2} e^{2 j \theta}+\cdots .
$$

At this level of approximation, we are permitted to use the results of Fourier analysis to determine the $E_{n}$ 's. Consequently, we have the explicit formula ${ }^{3}$

$$
E_{n}=\frac{\omega}{(2)_{n} \pi} \int_{0}^{2 \pi / \omega} \varepsilon\left(I_{1} \cos \omega t\right) e^{-n j \omega t} d t .
$$

Since these are voltage components generated by the nonlinear element as a result of the current whose amplitude is $I_{1}$, we can at this point introduce "vector impedances," $Z_{n}$, such that $E_{n}=Z_{n} I_{1}$. Hence,

$$
\varepsilon=Z_{0} I_{1}+Z_{1} I_{1} e^{j \theta}+Z_{2} I_{2} e^{2 j \theta}+\cdots
$$

where

$$
Z_{n}=\frac{\omega}{(2)_{n} \pi I_{1}} \int_{0}^{2 \pi / \omega} \mathcal{E}\left(I_{1} \cos \omega t\right) e^{-n i \omega t} d t
$$

or

$$
Z_{n}=\frac{1}{(2)_{n} I_{1}} \int_{0}^{2 \pi} \mathcal{E}\left(I_{1} \cos \theta\right) e^{-n j \theta} d \theta .
$$

In the latter expression, operators such as $d / d t$, occurring within $\varepsilon$, are to be replaced by equivalent operators $1 / \omega d / d \theta$, etc.

Clearly, the $Z$ 's (and $E$ 's) will be functions of $I_{1}$ and $\omega$. Since at later levels of approximation, $I_{1}$ varies slowly, we see that the $Z$ 's, too, will vary slowly, even though they are obtained under conditions that would indicate they are constant.

Let us now recast the above results in terms of our fullexponential notation. We introduce the following complex

\footnotetext{
${ }^{2}$ Whenever the current, $i$, is inserted in the nonlinear operator, real notation must be substituted for the complex notation because nonlinear operations erroneously intertangle real and imaginary parts. Thus, we must use $I_{1} \cos \theta$ for the above expression.

${ }^{3}$ In order to avoid writing out separately the zeroth Fourier coefficient, which has a troublesome factor of $1 / 2$ not contained in the other coefficients, we have used the symbol $(2)_{n}$ to indicate a quantity which is 2 when $n=0$ and unity otherwise.
}

variables which are mixtures of the $\phi_{n}$ 's and $n \theta$ 's that we used in the $\psi_{n}$ 's:

$$
\psi_{m n}=\phi_{m}+n j \theta
$$

In these terms, we write

$$
\varepsilon\left(e^{\phi_{1}} \cos \theta\right)=Z_{0} e^{\psi_{10}}+Z_{1} e^{\psi_{11}}+Z_{2} e^{\psi_{12}}+\cdots
$$

where the $Z$ 's now have the form

$$
Z_{n}=\frac{1}{(2)_{n}} \int_{0}^{2 \pi} \varepsilon\left(e^{\phi_{1}} \cos \theta\right) e^{-\psi_{1} m} d \theta .
$$

In this expression, $\phi_{1}$ is to be considered as a purely real constant.

Next, let us consider the second order terms that occur in $\varepsilon(i)$. These terms will arise from two sources. First, in addition to the dominant zero order term, $I_{1} e^{j \theta}$, we must also include the first-order terms, ${ }^{4} I_{2} e^{2 j \theta}, I_{3} e^{3 i \theta}$, etc. in our expression for $i$. Secondly, within the operator, $\varepsilon$, we must now take into account the fact that $I_{1}$ has first order variations with the time. This means, for example, that time derivatives must be permitted to act once upon $I_{1}$.

Since the terms $I_{2}, I_{3}$, etc., and the first-order variations of $I_{1}$ all represent perturbations in the current compared to the dominant term $I_{1}$, their contributions to $\mathcal{E}(i)$ are of second order. We may compute these contributions in the following way. First the complex expression

$$
i=I_{1} e^{j \theta}+I_{2} e^{2 i \theta}+I_{3} e^{3 i \theta} \ldots
$$

is put in real form. Then, since we have already computed the set of first-order terms corresponding to $\mathcal{E}\left(I_{1} \cos \theta\right)$, we may introduce the perturbation currents $I_{2}, I_{3}$, etc., one at a time, along with the dominant current, $I_{1}$, (considered constant) and compute the resulting changes. We also introduce $I_{1}$ by itself and compute the change caused by the operators within $\&$ acting upon the firstorder variation of $I_{1}$. Next, all of these changes are subjected to Fourier analysis to separate out the frequency contributions of each. In this Fourier analysis, $I_{1}, I_{\Sigma}, I_{3}$, etc. and the first-order variations of $I_{1}$ are all considered to be constant.

We now see that the above set of Fourier coefficients for the changes can be expressed in the form of a matrix as follows:

$$
\begin{aligned}
\tilde{Z}_{01} I_{1}+Z_{02} I_{2}+Z_{03} I_{3} & \cdots \\
& +\left(\tilde{Z}_{11} I_{1}+Z_{12} I_{2}+Z_{13} I_{3} \cdots\right) e^{j \theta} \\
& +\left(\widetilde{Z}_{21} I_{1}+Z_{22} I_{2}+Z_{23} I_{3} \cdots\right) e^{2 i \theta} \\
& +\left(\widetilde{Z}_{31} I_{1}+Z_{32} I_{2}+Z_{33} I_{3} \cdots\right) e^{3 j \theta} \\
& + \text { etc. }
\end{aligned}
$$

Thus the term, $Z_{02} I_{2}$, represents the zeroth Fourier coefficient of the change caused by the addition of the current component $I_{2}, Z_{12} I_{2}$ the first Fourier coefficient of this same change and so on. The terms $\widetilde{Z}_{01} I_{1}, \widetilde{Z}_{11} I_{1}$, etc.

\footnotetext{
${ }^{4}$ The blocking action of the capacitor in the circuit is such as to make $I_{0}$ of second order.
} 
represent the corresponding Fourier coefficients of the changes caused by the first-order variation of $I_{1}$ and so on.

We may now combine the vector $Z_{n}$ 's obtained earlier for the first-order terms for $\varepsilon(i)$ with these second-order matrix terms. To do this, we define the following $Z$ 's for the first column of the matrix $Z_{n 1}=Z_{n}+\widetilde{Z}_{n 1}$. Using this notation, we may write, to second order:

$$
\mathcal{E}(i)=\sum_{m, n} Z_{m n} I_{n} e^{j m \theta} .
$$

We thus see that we have succeeded in defining an impedance matrix which represents the behavior of nonlinear elements to second-order accuracy. It is a straightforward matter to evaluate the components of the matrix if we are given the operator $\varepsilon(i)$. It is readily verified that if the method of evaluation given above for nonlinear elements is used for a linear element, the resulting matrix will agree with the impedance matrices given earlier for linear elements.

Nonlinear elements, in contrast to linear elements, will have matrix terms lying off the diagonal of the matrix. These terms represent the cross-coupling among frequency components that is characteristic of nonlinear phenomena. It should be noticed that since we have taken into account second-order terms, the impedance matrix gives not only the transfer from the dominant frequency to the lesser frequencies, but also the return coupling from the lesser frequencies to the dominant frequency and the cross coupling among the lesser frequencies, as well as the transfer caused by variations of the dominant frequency.

The term, $Z_{11}$, of the impedance matrix is the usual nonlinear impedance of the describing function method and the method of equivalent linearization. It represents solely the reaction of the nonlinear element to the dominant current in producing a voltage at the dominant frequency.

We can express the above matrix results in terms of our full exponential notation as follows:

$$
\mathcal{E}(i)=\sum_{m n} Z_{m n} e^{\psi n m}
$$

Next, let us turn our attention to the third-order terms that occur in the nonlinear operator, $\varepsilon(i)$. In evaluating these terms, we must now take into account second-order variations of $I_{1}\left(e . g . d^{2} I_{1} / d t^{2}\right)$ and of $I_{2}, I_{3}$, etc. (since $I_{2}, I_{3}$, etc. are themselves of first order, their derivatives, $d I_{2} / d t, d I_{3} / d t$, etc. are such second-order variations). These variations will cause third-order changes to appear in $E(i)$; and if we evaluate these changes one at a time, and subject them to Fourier analysis, we may separate them out as third-order contributions to the previously defined impedance matrix.

However, this is not all. We must also take into account. the simultaneous occurrence of first-order terms, such as $I_{2}$ and $I_{3}$. These will cause third-order terms to appear in $\&(i)$. We cannot represent the Fourier coefficients of these terms by a second-rank matrix. Consequently, their effect cannot be simply added to the existing impedance matrix. Instead, we must introduce a third-rank impedance matrix to represent this higher-order cross-coupling.

The procedure to obtain progressively higher-order terms for $\varepsilon(i)$ is now clear. We must take into account successively higher-order variations in the expressions we use for $i$. Furthermore, we must take into account ever more complex combinations of the simultaneous occurrence of the lower-order terms. In representing the results, we must also introduce successively higher-rank $Z$ matrices.

It should be noted that in many cases, $I_{2}, I_{3}, I_{4}$, etc. will not all start off as first-order terms. Some will be firstorder, some will be second-order and so on. When this is the case, the notation must be modified accordingly. Furthermore, it may occur that integrations as well as differentiations, etc. will occur in $E(i)$. In this case also, the orders of magnitudes of the terms will be affected and the notation must be modified to take this into account.

In the nonlinear matrices we have obtained, just as in the linear ones, we still face the problem of eliminating the frequency derivatives if we are to develop an operational procedure which substitutes algebra for the complexities of solving differential equations (of course, we also need to eliminate the amplitude derivatives as well, but with the complex exponential notation, amplitude derivatives simply become the real part of the complex frequency derivatives).

\section{Analysis of the Complete Circuit}

Now that we have determined the frequency behavior of the individual components, let us consider the behavior of the complete circuit described by the equation

$$
L \frac{d i}{d t}+\frac{1}{C} \int i d t=-\varepsilon(i) .
$$

Having put

$$
i=e^{\psi_{0}}+e^{\psi_{2}}+e^{\psi_{2}}+e^{\psi_{3}} \cdots
$$

with $e^{\psi_{1}}$ dominating the other terms, we have found that

$$
L \frac{d i}{d t}=L p_{0} e^{\psi_{0}}+L p_{1} e^{\psi_{1}}+L p_{2} e^{\psi_{2}}+L p_{3} e^{\psi_{3}} \cdots
$$

and $^{5}$

$$
\begin{aligned}
\frac{1}{C} \int i d t & =\frac{Q}{C} \\
+ & \frac{1}{C}\left[1+\frac{d p_{1} / d t}{p_{1}^{2}}-\frac{d^{2} p_{1} / d t^{2}}{p_{1}^{3}}+\frac{3\left(d p_{1} / d t\right)^{2}}{p_{1}^{4}} \cdots\right] \frac{e^{\psi_{2}}}{p_{1}} \\
+ & \frac{1}{C}\left[1+\frac{d p_{2} / d t}{p_{2}^{2}}-\frac{d^{2} p_{2} / d t^{2}}{p_{2}^{3}}+\frac{3\left(d p_{2} / d t\right)^{2}}{p_{2}^{4}} \cdots\right] \frac{e^{\psi_{2}}}{p_{2}} \\
+ & \frac{1}{C}\left[1+\frac{d p_{3} / d t}{p_{3}^{2}}-\frac{d^{2} p_{3} / d t^{2}}{p_{3}^{3}}+\frac{3\left(d p_{3} / d t\right)^{2}}{p_{3}^{4}} \cdots\right] \frac{e^{\psi_{3}}}{p_{3}}
\end{aligned}
$$

etc.

${ }^{5}$ We have put $Q$ equal to the slowly varying do charge on the condenser. 
We have also found

$$
\begin{gathered}
\varepsilon(i)=+Z_{01} e^{\psi_{10}}+Z_{02} e^{\psi_{20}}+Z_{03} e^{\psi_{30}} \ldots \\
+Z_{11} e^{\psi_{11}}+Z_{12} e^{\psi_{21}}+Z_{13} e^{\psi_{31}} \ldots \\
+Z_{21} e^{\psi_{12}}+Z_{22} e^{\psi_{22}}+Z_{23} e^{\psi_{32}} \ldots \\
+Z_{31} e^{\psi_{13}}+Z_{32} e^{\psi_{23}}+Z_{33} e^{\psi_{33}} \ldots \\
\vdots \\
\vdots
\end{gathered}
$$

+ third- and higher-order terms.

When we place these expressions in the circuit equation, we face the problem of separating out the various frequency components. With the amplitudes and frequencies varying, this separation is to some extent arbitrary. However, since the $n \theta$ term is dominant in both

$$
\psi_{n}=\phi_{n}+n j \theta \text { and in } \psi_{m n}=\phi_{m}+n j \theta,
$$

a consistent method is to equate coefficients having the same values of $n$. This is equivalent to the usual method of equating coefficients of corresponding frequencies. If we do this, we obtain

$$
L p_{0} e^{\phi_{0}}+\frac{Q}{C}=-R e\left[Z_{01} e^{\phi_{1}}+Z_{02} e^{\phi_{2}}+Z_{03} e^{\phi_{3}} \cdots\right]
$$

+ third- and higher-order terms.

$$
\begin{aligned}
{\left[L p_{1}+\frac{1}{. p_{1} C}\left(1+\frac{d p_{1} / d t}{p_{1}^{2}} \cdots\right)\right] e^{\phi_{1}} } & \\
& =-\left[Z_{11} e^{\phi_{1}}+Z_{12} e^{\phi_{2}}+Z_{13} e^{\phi_{3}} \cdots\right]
\end{aligned}
$$

+third- and higher-order terms.

$$
\begin{aligned}
{\left[L p_{2}+\frac{1}{p_{2} C}\left(1+\frac{d p_{2} / d t}{p_{2}^{2}} \ldots\right)\right] e^{\phi_{2}} } & \\
& =-\left[Z_{21} e^{\phi_{1}}+Z_{22} e^{\phi_{2}}+Z_{23} e^{\phi_{3}} \cdots\right]
\end{aligned}
$$

+ third- and higher-order terms.

There will be similar equations for the higher harmonics.

It will be remembered that in these equations, $p_{n}=$ $\lambda_{n}+n j \omega$ where $\lambda_{n}=d \phi_{n} / d t$. The quantities $\omega$ and $e^{\theta_{2}}$ are of zero order, the quantities $Q, \lambda_{1}, e^{\theta_{2}}, e^{\theta_{3}}, \cdots$, are of first order,

$$
e^{\phi_{0}}=\frac{d Q}{d t}, \quad \frac{d \lambda_{1}}{d t}, \quad \lambda_{2}, \lambda_{3}, \cdots,
$$

are of second order,

$$
\lambda_{0}, \quad \frac{d^{2} \lambda_{1}}{d t^{2}}, \quad \frac{d \lambda_{2}}{d t}, \quad \frac{d \lambda_{3}}{d t} \cdots,
$$

are of third order, and so on. All of the $Z_{m n}$ 's are of first order, and to second-order accuracy, they may be considered to be independent of $e^{\phi_{2}}$, $e^{\phi_{0}}$, etc.

In the above equations, only the second equation, which is the one for the dominant current component, contains terms of zero order. If we were to ignore all but these zero-order terms, we should of course abtain the result

$$
p_{1}=\frac{j}{\sqrt{L C}}, \quad 0=e^{\phi_{0}}=e^{\phi_{2}}=e^{\phi_{3}}
$$

etc; that is, that only the dominant oscillation is present and it has the frequency, $1 / \sqrt{L C}$.

However, if we consider both zero-order and first-order terms, this equation says that the frequency of the dominant oscillation becomes

$$
p_{1}=\frac{1}{\sqrt{L C}}\left(j-\frac{Z_{11}}{2} \sqrt{\frac{C}{L}}\right) \text { or } \quad \lambda_{1}=-\frac{Z_{11}}{2 L} .
$$

Since $Z_{11}$ contains both real and imaginary parts, we see that the nonlinear element acts to shift both the exponential and the trigonometric frequencies of the current, and that these frequencies are variable with the time. We also find that, to first order, the nonlinear element induces a charge,

$$
Q=-Z_{01} e^{\phi_{1}}=-Z_{01} I_{1},
$$

on the capacitor and that it generates the harmonic components

$$
\begin{aligned}
& e^{\phi_{2}}=\frac{2 j}{3} \sqrt{\frac{C}{L}} Z_{21} e^{\phi_{1}} \quad \text { or } \quad I_{2}=\frac{2 j}{3} \sqrt{\frac{C}{L}} Z_{21} I_{1}, \\
& e^{\phi_{3}}=\frac{3 j}{8} \sqrt{\frac{C}{L}} Z_{31} e^{\phi_{1}} \quad \text { or } \quad I_{3}=\frac{3 j}{8} \sqrt{\frac{C}{L}} Z_{31} I_{1},
\end{aligned}
$$

and so on.

Now we come to the decisive step of considering the second-order terms. So far, we have used only the algebraic manipulations such as are characteristic of operational methods. However, we are now confronted with the second-order derivative

$$
\frac{d p_{1}}{d t}=\frac{d \lambda_{1}}{d t}=\frac{d^{2} \phi_{1}}{d t^{2}}
$$

Our method of meeting this situation is as follows. It is readily verified, that to second-order accuracy, we may use our first-order result, $\lambda_{1}=-Z_{11} / 2 L$ to evaluate $d p_{1} / d t$. In so doing, we must remember that $Z_{11}$ is a function of $\phi_{1}$ or $I_{1}$ and, since $\phi_{1}$ varies with the time, $Z_{11}$ has a time derivative. We have the explicit Fourier formula,

$$
Z_{11}=\frac{e^{-\phi_{1}}}{\pi} \int_{0}^{2 \pi} \varepsilon\left(e^{\phi_{1}} \cos \theta\right) e^{-i \theta} d \theta
$$

which gives $Z_{11}$ as a function of $\phi_{1}$. We may differentiate this formula with respect to $\phi_{1}$, but in so doing we must remember that in this formula we have used the local prerogative of choosing a time base such that $\phi_{1}$ is purely real. In reality, over extended periods of time, $\phi_{1}$ will become complex. The $\phi_{1}$ in the formula above is then the 
real part of the complex $\phi_{1}$. Bearing this in mind, we have

$$
\begin{aligned}
\frac{d p_{1}}{d t}=\frac{d \lambda_{1}}{d t}=-\frac{1}{2 L} & \frac{d Z_{11}}{d t} \\
& =-\frac{1}{2 L} \frac{d Z_{11}}{d \phi_{1}} \operatorname{Re}\left(\frac{d \phi_{1}}{d t}\right)=\frac{\operatorname{Re}\left(Z_{11}\right)}{4 L^{2}} \frac{d Z_{11}}{d \phi_{1}} .
\end{aligned}
$$

This is an algebraic expression giving $d p_{1} / d t$ in terms of the $Z$ matrix component, $Z_{11}$, which we can readily calculate. This may be written in the form

$$
\frac{1}{p_{1}^{2}} \frac{d p_{1}}{d t}=-\frac{C}{4 L} \operatorname{Re}\left(Z_{11}\right) \frac{d Z_{11}}{d \phi_{1}}=-\frac{I_{1} C}{4 L} \operatorname{Re}\left(Z_{11}\right) \frac{d Z_{11}}{d I_{1}} .
$$

This result is of great importance to us because it furnishes us the basis for an answer to the second goal which we posed earlier; namely, the establishment of a workable method of operational analysis which would permit us to use algebraic manipulation in treating the slowly varying amplitudes and frequencies that occur in nonlinear analysis. This result allows us to replace the unknown frequency derivative, $d p_{1} / d t$, by an expression which can be calculated directly from the impedance matrix of the nonlinear elements. In a similar way, we find from the first-order result,

$$
\begin{aligned}
e^{\phi_{2}}=\frac{2 j}{3} \sqrt{\frac{C}{L}} Z_{21} e^{\phi_{1}} \quad \text { that } \frac{d \phi_{2}}{d t}= & \frac{d \phi_{1}}{d t} \\
+ & +\frac{1}{Z_{21}} \frac{d Z_{21}}{d t} \\
& =-\frac{Z_{11}}{2 L}-\frac{\operatorname{Re}\left(Z_{11}\right)}{2 L Z_{21}} \frac{d Z_{21}}{d \phi_{1}}
\end{aligned}
$$

or

$$
p_{2}=\frac{2 j}{\sqrt{L C}}-\frac{Z_{11}}{2 L}-\frac{\operatorname{Re}\left(Z_{11}\right)}{2 L Z_{21}} \frac{d Z_{21}}{d \phi_{1}}
$$

We also have

$$
p_{3}=\frac{3 j}{\sqrt{L C}}-\frac{Z_{11}}{2 L}-\frac{\operatorname{Re}\left(Z_{11}\right)}{2 L Z_{31}} \frac{d Z_{31}}{d \phi_{1}}, \text { etc. }
$$

In linear analysis, impedances never depend upon current amplitudes, so the terms $d Z_{n 1} / d \phi_{1}$ never arise. Also cross-coupling terms like $Z_{21}, Z_{31}$, etc. never arise. However, when nonlinear elements are present, they thrust themselves into the impedance matrices of linear elements by these terms.

Returning to our task of solving the circuit equations, we see that if we use the above result for $d p_{1} / d t$, then by purely algebraic methods we may solve for the frequency, $p_{1}$ to second-arder accuracy.

$$
\begin{aligned}
p_{1}=\frac{1}{\sqrt{L C}}\left[j-\frac{Z_{11}}{2} \sqrt{\frac{C}{L}}\right. & +\frac{C}{L}\left(\frac{j Z_{11}^{2}}{8}+j \frac{\operatorname{Re}\left(Z_{11}\right)}{8} \frac{d Z_{11}}{d \phi_{1}}\right. \\
& \left.\left.+\frac{1}{3} Z_{21} Z_{12}+\frac{3}{16} Z_{31} Z_{13} \cdots\right)\right] .
\end{aligned}
$$

We may also use similar procedures to express the current components $I_{0}, I_{2}, I_{3}$, etc. in terms of $I_{1}$. Thus, we have

$I_{0}=e^{\phi_{0}}=\frac{1}{2} \frac{C}{L} \operatorname{Re}\left(Z_{11}\right) \operatorname{Re}\left[\left(Z_{01}+\frac{d Z_{01}}{d \phi_{1}}\right) e^{\phi_{2}}\right]$,

$$
\begin{aligned}
I_{2}=e^{\phi_{2}} & =e^{\phi_{1}}\left[\frac{2 j}{3} \sqrt{\frac{C}{L}} Z_{21}+\frac{C}{L}\left(\frac{5}{18} Z_{11} Z_{21}\right.\right. \\
& \left.\left.+\frac{5}{18} \operatorname{Re}\left(Z_{11}\right) \frac{d Z_{21}}{d \phi_{1}}-\frac{4}{9} Z_{21} Z_{22}-\frac{1}{4} Z_{31} Z_{23} \ldots\right)\right],
\end{aligned}
$$

etc.

It is now clear how we may proceed to third-order and higher approximations. At each step we use the same basic procedure of computing all of the necessary frequency derivatives from the results of the preceding step. Then, purely by algebraic manipulation, we may solve for the frequency of the dominant component, $I_{1}$ and for the amplitudes (and frequencies, if desired) of the lesser components $I_{0}, I_{2}, I_{3}$, etc. These will all be expressed in terms of $I_{1}$ and of the $Z$ matrices which characterize the nonlinear elements.

Suppose that we have carried these successive approximations to a point which gives us the appropriate accuracy that we desire. We now face the problem of finding the explicit dependence of $\phi_{1}$ or $I_{1}$ upon the time. Knawing this, the time dependence of all the order quantities is easily obtained. So far, we have been very careful to obtain results which are uniformly valid for all time. We have avoided the usual perturbation procedure which develops successive approximations explicitly in terms of the time. As we saw earlier, such a shortsighted procedure leads to the inconsistency of expressing the longterm behavior of the system in terms of power series which are short-term modes of expression.

Let us focus our attention on the solution we have obtained for $p_{1}$. It is expressed entirely in terms of the components of the $Z$ matrices. These, in turn, are functions of $\phi_{0}, \phi_{1}, \phi_{2}, \phi_{3}$, etc. However, since we know $\phi_{0}$, $\phi_{2}, \phi_{3}$, etc., in terms of $\phi_{1}$, we can express $p_{1}$ entirely in terms of $\phi_{1}$. It is true that the relations between $\phi_{0}, \phi_{2}$, $\phi_{3}$, etc., and $\phi_{1}$ are expressed in terms of $Z$ matrices which contain $\phi_{0}, \phi_{2}, \phi_{3}$, etc. However, it is readily verified that successive interactions will produce expressions for $\phi_{0}$, $\phi_{2}, \phi_{3}$, etc., that contain only $\phi_{1}$. Consequently, we can obtain an expression for $p_{1}$ which contains only $\phi_{1}$. This is readily accomplished if we are satisfied with secondorder accuracy, because at this level the matrices are all independent of $\phi_{0}, \phi_{2}, \phi_{3}$, etc.

Now that we are setting out to obtain the long term behavior of $\phi_{1}$ we are again confronted with the fact that $\phi_{1}$ will become complex and in our impedance matrices we have used a local time base which makes $\phi_{1}$ real. As pointed out previously, this means that the $\phi_{1}$ in the matrices is the real part of the complex $\phi_{1}$. At this point, we separate $\phi_{1}$ into real and imaginary parts; thus $\phi_{1}=$ $\phi_{r}+j \phi_{j}$. Consequently, we have

$$
p_{1}=\frac{d \phi_{r}}{d t}+j \frac{d \phi_{i}}{d t}+\frac{j}{\sqrt{L C}}
$$

Let us correspondingly designate by $p_{r}+j p_{i}$ the expression which we obtained above for $p_{1}$ in terms of the $Z$ matrices. We have just seen that these matrices are 
functions of the real part of $\phi_{1}$, i.e., of $\phi_{r}$. Consequently, we may write

$$
p_{r}=p_{r}\left(e^{\phi_{r}}\right) \text { and } p_{i}=p_{i}\left(e^{\phi_{r}}\right) .
$$

Furthermore, we may separate the equation

$$
p_{1}=\frac{d \phi_{1}}{d t}+\frac{j}{\sqrt{L C}}
$$

into real and imaginary parts as follows

$$
\begin{aligned}
\frac{d \phi_{r}}{d t} & =p_{r}\left(e^{\phi_{r}}\right) \\
\frac{d \phi_{j}}{d t} & =p_{i}\left(e^{\phi_{r}}\right)-\frac{1}{\sqrt{L C}} .
\end{aligned}
$$

The first of these equations is the all-important equation for the time variation of the amplitude, $\left|I_{1}\right|$, with respect to $t$, as we may see when we write it in the form

$$
\frac{1}{\left|I_{1}\right|} \frac{d\left|I_{1}\right|}{d t}=p_{r}\left(\left|I_{1}\right|\right)
$$

Fortunately, this equation can always be solved explicitly because the variables are separable.

$$
t=\int \frac{d\left|I_{1}\right|}{\left|I_{1}\right| p_{r}\left(\left|I_{1}\right|\right)}
$$

We notice that the solution gives $t$ as a function of $\left|I_{1}\right|$ instead of the usual form which gives $\left|I_{1}\right|$ as a function of $t$. However, a moment's reflection shows that this result is a necessary consequent of our goal to establish a method which gives the above relationship in a form having long-term validity, a goal which we have now accomplished. The reason for this is as follows. In autonomous nonlinear circuits, the fundamental relationships among the variables do not involve the time explicitly. Rather the relationships are among the dependent variables themselves. When a dominant oscillation is present, it is the amplitude of this oscillation which determines the state of the circuit. Consequently, it is appropriate to express the state equations of the circuit in terms of this variable so that they will have uniform validity. This we have done. It then follows that all the other variables (including the time) will finally be expressed in terms of this principal state variable.

The "instantaneous" frequency of the dominant oscillation is given by $p_{i}=p_{j}\left(\left|I_{1}\right|\right)$. If we want the "instantaneous" phase angle $\phi_{j}$, we first write

$$
\frac{d \phi_{i}}{d \phi_{r}}=\frac{p_{i}\left(e^{\phi_{r}}\right)-\frac{1}{\sqrt{L C}}}{p_{r}\left(e^{\phi_{r}}\right)}
$$

We then solve to give

$$
\phi_{i}=\int \frac{p_{j}\left(e^{\phi_{r}}\right)-\frac{1}{\sqrt{L C}}}{p_{r}\left(e^{\phi_{r}}\right)} d \phi_{r}
$$

or

$$
\phi_{i}=\int \frac{p_{i}\left(\left|I_{1}\right|\right)-\frac{1}{\sqrt{L C}}}{\left|I_{1}\right| p_{r}\left(\left|I_{1}\right|\right)} d\left|I_{1}\right| \text {. }
$$

We have already given expressions for $I_{0}, I_{2}, I_{3}$, etc. in terms of $I_{1}$.

In principle, we can always invert the above relationship between $t$ and $I_{1}$. If we do this, we can express all of the amplitudes and frequencies directly in terms of the time. In so doing, we must be careful not to lose the long term validity of the results.

\section{A More General View of the Operational Procedure}

So far we have confined our attention to the equation,

$$
L \frac{d i}{d t}+\frac{1}{C} \int i d t=\varepsilon(i) .
$$

Now let us examine the more general equation

$$
\begin{aligned}
\cdots A_{3} \frac{d^{3} i}{d t^{3}}+A_{2} \frac{d^{2} i}{d t^{2}}+A_{1} & \frac{d i}{d t} \\
& +A_{0} i+A_{-1} \int i d t \cdots=\varepsilon(i) .
\end{aligned}
$$

In treating this equation, we shall subject our analysis to the following restrictions. First, since we are dealing with oscillatory solutions, we shall require that the basic linear circuit, represented by the operator on the left side of the above equation, have an undamped oscillation of frequency $\omega$. This means that the expression

$$
\cdots A_{3} p^{3}+A_{2} p^{2}+A_{1} p+A_{0}+\frac{A_{-1}}{p} \cdots
$$

has a root $p=j \omega$. Secondly, we restrict our attention to those patterns of circuit behavior in which an oscillation having a frequency near $\omega$ is dominant.

We again express $i$ in the form

$$
i=e^{\psi_{0}}+e^{\psi_{1}}+e^{\psi_{2}} \cdots
$$

where

$$
\psi_{n}=\phi_{n}+j n \theta
$$

and

$$
p_{n}=\frac{d \psi_{n}}{d t}=\lambda_{n}+j n \omega
$$

with

$$
\lambda_{n}=\frac{d \phi_{n}}{d t} \text { and } \omega=\frac{d \theta}{d t}
$$

Furthermore, we express $\varepsilon(i)$ in terms of the impedance matrices.

Now it is readily verified that to first-order accuracy

$$
\lambda_{1}=-\frac{j \omega Z_{11}}{\cdots 3 A_{3}(j \omega)^{3}+2 A_{2}(j \omega)^{2}+A_{1}(j \omega)+A_{-1}\left(\frac{1}{j \omega}\right) \ldots} .
$$


At this level of accuracy, the terms $A_{3}(j \omega)^{3}, A_{2}(j \omega)^{2}$, etc. are just the basic impedances of the corresponding linear operators. Thus, if we put $Z_{n}^{\neq}=A_{n}(j \omega)^{n}$ and designate the weighted sum of these impedances by $\bar{Z}_{\mathrm{Lin} .}=\sum_{n} n Z_{n}^{\neq}$, we have

$$
\lambda_{1}=-\frac{j \omega Z_{11}}{\bar{Z}_{\mathrm{Lin}}}
$$

If we go to second-order accuracy, we encounter the term $\left(1 / p_{1}^{2}\right) d p_{1} / d t$ in the linear operators. Since

$$
\frac{d p_{1}}{d t}=\frac{d \lambda_{1}}{d t}=\lambda_{1} \frac{d \lambda_{1}}{d \phi_{1}}=\lambda_{1} I_{1} \frac{d \lambda_{1}}{d I_{1}}
$$

we may obtain the following explicit expression for this term

$$
\frac{1}{p_{1}^{2}} \frac{d p_{1}}{d t}=-\frac{\operatorname{Re}\left(Z_{11}\right) I_{1}}{\bar{Z}_{\mathrm{Lin} .}^{2}} \frac{d Z_{11}}{d I_{1}}
$$

Thus, for each of the linear terms, we have the operational expression

$$
A_{n} p_{1}^{n}\left[1-n(n-1) \frac{\operatorname{Re}\left(Z_{11}\right) I_{1}}{\bar{Z}_{\mathrm{Lin} .}^{2}} \frac{d Z_{11}}{d I_{1}}\right]
$$

at the $(1,1)$ location in the impedance matrix for this linear term. This expression replaces the usual value, $A_{n} p_{1}^{n}$, commonly used in linear operational procedures. This modification of the usual value represents the effect of nonlinear interaction within the circuit upon the impedance of this linear term.

For the other current components, we find that, to first order,

$$
I_{m}=e^{\phi_{m}}=-\frac{Z_{m} e^{\phi_{2}}}{\sum_{n} A_{n}(j m \omega)^{n}}=-\frac{Z_{m 1} I_{1}}{\sum_{n} m^{n} Z_{n}^{\mp}} .
$$

When we go to second order, the impedance term at the $(m, m)$ location in the impedance matrix for $A_{n} d^{n} i / d t^{n}$ is given by

$$
A_{n}\left[(j m \omega)^{n}+n(j m \omega)^{n-1} \frac{d \phi_{m}}{d t}\right]=m^{n} Z_{n}^{\neq}\left(1+\frac{n}{m j \omega} \frac{d \phi_{m}}{d t}\right) .
$$

However, from the first-order expressions given above, we find that

$$
\frac{1}{j \omega} \frac{d \phi_{m}}{d t}=-\frac{Z_{11}+\frac{\operatorname{Re}\left(Z_{11}\right)}{Z_{m 1}}}{\bar{Z}_{\mathrm{Lin}}} \frac{d Z_{m 1}}{d \phi_{1}} .
$$

Consequently, the impedance term at the $(m, m)$ location in the matrix for $A_{n} d^{n} i / d l^{n}$ is given by

$$
m^{n} Z_{n}^{\neq}\left[1-\frac{n}{m} \cdot \frac{Z_{11}+\frac{\operatorname{Re}\left(Z_{11}\right)}{Z_{m 1}} \frac{d Z_{m 1}}{d \phi_{1}}}{\bar{Z}_{\mathrm{Lin} .}}\right]
$$

instead of the usual value, $m^{n} Z_{n}^{\ngtr}$.

Using these modified expressions for the impedance matrices of the linear terms and the full second-order matrix, $Z_{m n}$ for $\mathcal{E}(i)$, we may solve by algebraic methods for the complete set of frequencies and currents, all in terms of the dominant current amplitude. These results will be valid to second order and will exhibit the full set of nonlinear interactions that are appropriate at this level of accuracy.

If desired, the solution can be carried to higher orders of accuracy by using straightforward extension of the procedures we have given above. When the desired local accuracy is achieved, then just as in the case of the simple $L C$ circuit, the expression for the frequency $p_{1}$ can be separated into real and imaginary parts and the real part can be integrated to give an explicit relationship between the dominant current component and the time. From this, all the other frequencies and amplitudes may be found as functions of the time.

We may also generalize the above analysis in another way. So far we have assumed that all of the principal circuit elements are linear and that the nonlinear elements act as perturbing elements in the circuit. The linear elements are characterized by impedance matrices having only diagonal terms. These terms are, of course, of zero order. The nonlinear elements are characterized by impedance matrices having first-order terms in the column of the dominant oscillation and second-order terms elsewhere.

Suppose that we were to combine a principal linear element with a perturbing nonlinear element, and to treat the combination as a single element. Such a "moderately nonlinear" element would have zero-order terms on the diagonal, first-order terms in the column of the dominant oscillation, and second-order terms elsewhere. It is now immaterial to us whether the element is a combination of a linear and a nonlinear element or is a "moderately nonlinear" element in its own right so long as these criteria for orders of magnitude are fulfilled.

If we re-examine our method of analysis, we see that we can treat equally well circuits containing such "moderately nonlinear" elements. In fact, the circuit can consist entirely of such elements. Typical of such elements would be inductances and capacitances which show moderate departures from linearity. However, the concepts are much broader than these simple examples would imply.

\section{EXAMPLES}

Let us now consider a few examples to illustrate the methods we have presented here. It is instructive to treat. the same examples which we solved earlier by the classical perturbation procedure so that we may compare the relative merits of the two procedures.

First, let us consider the case of perturbing elements consisting of both a linear capacitor, $C^{\prime}$, and a linear resistor, $R$. To second order, the impedance matrix of this combination is simply

$$
Z_{m n}=\delta_{m n}\left(R+\frac{1}{p_{n} C^{\prime}}\right) .
$$


The corresponding circuit equations are

$$
\begin{aligned}
& p_{0} L I_{0}+\frac{Q}{C}=-\left(I_{0} R+\frac{Q}{C^{\prime}}\right) \\
& p_{1} L I_{1}+\frac{I_{1}}{p_{1} C}\left(1+\frac{1}{p_{1}^{2}} \frac{d p_{1}}{d t}\right)=-I_{1}\left(R+\frac{1}{C^{\prime} p_{1}}\right), \\
& p_{2} L I_{2}+\frac{I_{2}}{p_{2} C}\left(1+\frac{1}{p_{2}^{2}} \frac{d p_{2}}{d t}\right)=-I_{2}\left(R+\frac{1}{C^{\prime} p_{2}}\right),
\end{aligned}
$$

etc.

To zero order, these equations give

$$
p_{1}=j \omega, \quad 0=I_{0}=I_{2}, \quad \text { etc. }
$$

To first order, they give

$$
p_{1}=j \omega\left(1+\frac{j R}{2} \sqrt{\frac{C}{L}}+\frac{1}{2} C_{C^{\prime}}^{C}\right), \quad 0=I_{0}=I_{2} \text {, etc. }
$$

From this latter result, we find $d p_{1} / d t=0 p_{2}=2 p_{1}$, etc. Consequently, to second order

$$
\begin{gathered}
\ddot{p}_{1}=j \omega\left[1+\frac{j R}{2} \sqrt{\frac{C}{L}}+\frac{1}{2} \frac{C}{C^{\prime}}-\frac{R^{2} C}{8 L}-\frac{1}{8}\left(\frac{C}{C^{\prime}}\right)^{2}\right] \\
0=I_{0}=I_{2} \text { etc. }
\end{gathered}
$$

If we are satisfied with this level of local accuracy, we may terminate these calculations and determine the long term behavior of the system. Separating the frequency, $p_{1}$, into real and imaginary parts and setting them equal to the derivatives of the corresponding phase angles, $d \phi_{r} / d t$ and $d \phi_{i} / d t+\omega$, we find

$$
\frac{d \phi_{r}}{d t}=-\frac{R}{2 L} \frac{d \phi_{i}}{d t}=\omega\left[\frac{1}{2} \frac{C}{C^{\prime}}-\frac{1}{8}\left(\frac{C}{C^{\prime}}\right)^{2}-\frac{R^{2} C}{8 L}\right]
$$

or

$\phi_{r}=-\frac{R t}{2 L}+$ const.,$\quad \phi_{i}=\omega t\left[\frac{1}{2} \frac{C}{C^{\prime}}-\frac{1}{8}\left(\frac{C}{C^{\prime}}\right)^{2}-\frac{R^{2} C}{8 L}\right]$

or

$$
i=I^{0} e^{-R t / 2 L} \cos \omega t\left[1+\frac{1}{2} \frac{C}{C^{\prime}}-\frac{1}{8}\left(\frac{C}{C^{\prime}}\right)^{2}-\frac{R^{2} C}{8 L}\right] .
$$

This result should be compared to the corresponding solution given by the classical perturbation procedure,

$$
\begin{aligned}
i=I^{0}\left\{\left[1-\frac{\omega t}{2}(R\right.\right. & \left.\left.\sqrt{\frac{C}{L}}\right)+\frac{\omega^{2} t^{2}}{8}\left(\frac{R^{2} C}{L}-\frac{C^{2}}{C^{\prime 2}}\right)\right] \cos \omega t \\
& \left.+\frac{\omega t}{2}\left[-\frac{C}{C^{\prime}}+\frac{1}{4}\left(\frac{C}{C^{\prime}}\right)^{2}-\frac{R^{2} C}{4 L}\right] \sin \omega t\right\}
\end{aligned}
$$

and to the exact solution,

$$
i=I^{0} e^{-R t / 2 L} \cos \omega t \sqrt{1+\frac{C}{C^{\prime}}-\frac{R^{2} C}{4 L}} .
$$

We see that the present method correctly predicts the long-term behavior of the system as an exponentially damped oscillation, whereas the classical method, at the same level of approximation, predicts a parabolically growing oscillation.
This example, in spite of its extreme simplicity, demonstrates how much closer the present method is to the usual procedure of solving linear equations than is the classical procedure and how experience gained from the usual procedure for linear equations can be used in the present method. Thus, in our solution above, we have used iteration procedures to accomplish the algebraic manipulations. However, we see that here the algebra is in fact so simple that iteration is not only unnecessary, it is undesirable. We can solve in the same way that is usually used for linear equations. Thus, for the dominant frequency, we have

$$
p_{1}=j \omega\left[\sqrt{1+\frac{C}{C^{\prime}}+\frac{1}{p_{1}^{2}} \frac{d p_{1}}{d t}-\frac{R^{2} C}{4 L}}-\frac{j R}{2} \sqrt{\frac{C}{L}}\right] .
$$

If we use the first-order version of this expression, we find that $d p_{1} / d t=0$, so that the above expression becomes

$$
p_{1}=j \omega\left[\sqrt{1+\frac{C}{C^{\prime}}-\frac{R^{2} C}{4 L}}-j \frac{R}{2} \sqrt{\frac{C}{L}}\right] .
$$

This is, of course, the correct result and if we integrate to obtain the long-term behavior, that too, will be correct. This close parallel does not exist with the classical procedure.

Let us now turn to the electrical circuit equivalent of the van der Pol equation

$$
L \frac{d i}{d t}+\frac{1}{C} \int i d t=R i\left(1-\frac{\beta i}{3}\right)^{2} .
$$

It is readily verified that only the odd harmonics are of interest in the solution, so we shall restrict our matrices to these components. The impedance matrix of the perturbing element on the right side is, to second order:

$$
\begin{gathered}
Z_{11}=-R\left(1-\frac{\beta I_{1}^{2}}{4}\right) \quad Z_{13}=\frac{R \beta I_{1}^{2}}{4} \quad Z_{15}=0 \cdots \\
Z_{31}=\frac{R \beta I_{1}^{2}}{12} \quad Z_{33}=-R\left(1-\frac{\beta I_{1}^{2}}{2}\right) \quad Z_{35}=\frac{R \beta I_{1}^{2}}{4} \cdots \\
Z_{51}=0 \quad Z_{53}=\frac{R \beta I_{1}^{2}}{4} \quad Z_{55}=-R\left(1-\frac{\beta I_{1}^{2}}{2}\right) \cdots .
\end{gathered}
$$

Also to second order, the circuit equations are

$$
p_{1} L I_{1}+\frac{I_{1}}{p_{1} C}\left(1+\frac{1}{p_{1}^{2}} \frac{d p_{1}}{d t}\right)
$$

$$
=R\left(1-\frac{\beta I_{1}^{2}}{4}\right) I_{1}-\frac{R \beta I_{1}^{2} I_{3}}{4}
$$

$p_{3} L I_{3}+\frac{I_{3}}{p_{3} C}\left(1+\frac{1}{p_{3}^{2}} \frac{d p_{3}}{d t}\right)$

$$
=-\frac{R \beta I_{1}^{3}}{12}+R\left(1-\frac{\beta I_{1}^{2}}{2}\right) I_{3}-\frac{R \beta I_{1}^{2} I_{5}}{4}
$$

$p_{5} L I_{5}+\frac{I_{5}}{p_{5} C}\left(1+\frac{1}{p_{5}^{2}} \frac{d p_{5}}{d t}\right)-\frac{R \beta I_{1}^{2} I_{3}}{4}+R\left(1-\frac{\beta I_{1}^{2}}{2}\right) I_{5} \cdots$

$$
\vdots \quad \vdots \quad \vdots \quad \vdots
$$


If we solve these equations to first order, we find

$$
\begin{aligned}
& p_{1}=j \omega+\frac{R}{2 L}\left(1-\frac{\beta I}{4}\right) \\
& I_{3}=\frac{j}{32} \sqrt{\frac{C}{L}} R \beta I_{1}^{3} \\
& I_{5}=0 .
\end{aligned}
$$

Using this result, we find

$$
\frac{1}{p_{1}^{2}} \frac{d p_{1}}{d t}=\frac{R^{2} C}{8 L} \beta I_{1}^{2}\left(1-\frac{\beta I_{1}^{2}}{4}\right)
$$

and

$$
p_{3}=3 j \omega+\frac{3 R}{2 L}\left(1-\frac{\beta I_{1}^{2}}{4}\right), \quad p_{5}=5 j \omega
$$

To second order, we find

$$
\begin{gathered}
p_{1}=j \omega+\frac{R}{2 L}\left(1-\frac{\beta I_{1}^{2}}{4}\right)+j \frac{\omega R^{2}}{8} \frac{C}{L}\left(3-\frac{\beta I_{1}^{2}}{4}\right)\left(1-\frac{\beta I_{1}^{2}}{4}\right) \\
I_{3}=j \frac{R}{32} \sqrt{\frac{C}{L}} \beta I_{1}^{3}\left[1+j \frac{R}{4} \sqrt{\frac{C}{L}}\left(1-\frac{\beta I_{1}^{2}}{4}\right)\right] \\
I_{5}=-\frac{5}{3072} \frac{R^{2} C}{L} \beta^{2} I_{1}^{5} .
\end{gathered}
$$

Again, if we are satisfied with second-order accuracy, we may terminate these calculations and obtain the longterm behavior of the system. If we separate the frequency, $p_{1}$, into its real and imaginary parts and equate them to the corresponding derivatives of the phase angles $d \phi_{r} / d t$ and $d \phi_{i} / d t+\omega j$ we find (using the notation $e^{\phi r}=I_{1}$ )

$$
\begin{gathered}
\frac{d \phi_{r}}{d t}=\frac{1}{I_{1}} \frac{d I_{1}}{d t}=\frac{R}{2 L}\left(1-\frac{\beta I_{1}^{2}}{4}\right) \\
\frac{d \phi_{i}}{d t}=\frac{\omega}{8} R^{2} \frac{C}{L}\left(3-\frac{\beta I_{1}^{2}}{4}\right)\left(1-\frac{\beta I_{1}^{2}}{4}\right) .
\end{gathered}
$$

These can be integrated to give

$$
\log \frac{I_{1}}{I^{0}} \sqrt{\frac{1-\frac{\beta I^{0^{2}}}{4}}{1-\frac{\beta I_{1}^{2}}{4}}}=\frac{R t}{2 L}
$$

where $I^{0}$ is the value of $I_{1}$ when $\dot{t}=0$, and

$$
\phi_{i}=\frac{R}{4} \sqrt{\frac{C}{L}}\left[\log \frac{I_{1}}{I^{0}}-\frac{\beta I_{1}^{2}}{8}+\frac{\beta I^{0^{2}}}{8}\right]+\phi^{0}
$$

with $\phi^{0}$ being the value of $\phi_{i}$ when $t=0$.

The first relation can be inverted to give

$$
I_{1}=\frac{I^{0}}{\sqrt{\left(1-\frac{\beta I^{0^{2}}}{4}\right) e^{-R t / 2 L}+\frac{\beta I^{0^{2}}}{4}}}
$$

as the slowly varying amplitude of the dominant oscillation and

$\frac{R}{8} \sqrt{\frac{C}{L}} \log \left[\left(1-\frac{\beta I^{02}}{4}\right) e^{-R t / 2 L}+\frac{\beta I^{02}}{4}\right]$

$$
-\frac{\left(1-\frac{\beta I^{02}}{4}\right)\left(1-e^{-R t / 2 L}\right)}{\left(1-\frac{\beta I^{02}}{4}\right) e^{-R t / 2 L}+\frac{\beta I^{0^{2}}}{4}}+\phi^{0}
$$

as its phase angle. Correspondingly, we find

$$
\frac{R}{32} \sqrt{\frac{C}{L}} \beta I_{1}^{3}
$$

as the amplitude of the third harmonic and

$$
\frac{\pi}{2}+\frac{R}{4} \sqrt{\frac{C}{L}}\left(1-\frac{\beta I_{1}^{2}}{4}\right)
$$

as its phase angle. The fifth harmonic has an amplitude

$$
\frac{5}{3072} R^{2} \frac{C}{L} \beta^{2} I_{1}^{5}
$$

and a phase angle $\pi / 2$.

In a global fashion, this solution gives the full sweep of the behavior of the Van der Pol equation, subject only to the restriction that the perturbations remain perturbations, i.e., that too large values of the initial $I^{0}$ be avoided.

\section{CONCLUSION}

We have shown that the classical perturbation procedure for treating nonlinear systems leads to solutions expressed as Fourier-like series with slowly varying coefficients. These slowly varying coefficients contain the information about the long-term behavior of the system. Inconsistently, the classical procedure expresses these coefficients as power series, a mode of expression which has notoriously poor long-term validity.

We have presented an operational procedure for treating oscillations having slowly variable amplitudes and frequencies. We have also presented an extension of the usual impedance concept which permits us to express the frequency characteristics of both linear and nonlinear elements when oscillations with many frequencies are present simultaneously and when these oscillations vary in both frequency and amplitude. Using these methods, we have devised a perturbation procedure for computing the behavior of systems to any order of accuracy, using only the algebraic processes which are characteristic of operational procedures. The procedure avoids expressing its results in terms of the local time. Instead, it expresses them in terms of the fundamental characteristics of the oscillations that are present in the system. As a consequence, the final solutions have the much desired longterm validity and they may be used to obtain asymptotic estimates of the behavior of the system. The method is able to treat systems containing nonlinear perturbing elements and elements which we have described as moderately nonlinear.

By means of examples, we show that it is a straightforward process to treat systems to second-order accuracy. This level of accuracy covers a large number of the intercoupling effects that characterize the more sophisticated nonlinear behavior. 